\title{
Persistent mutant oncogene specific T cells in two patients benefitting from anti-PD-1
}

Kellie N. Smith ${ }^{1,2+}$, Nicolas J. Llosa ${ }^{1,2+}$, Tricia R. Cottrell ${ }^{1,2,3}$, Nicholas Siegel ${ }^{1,2}$, Hongni Fan ${ }^{1,2}$, Prerna Suri ${ }^{1,2}$, Hok Yee Chan ${ }^{1,2}$, Haidan Guo ${ }^{1,2}$, Teniola Oke ${ }^{1,2}$, Anas H. Awan ${ }^{1,2}$, Franco Verde ${ }^{4}$, Ludmila Danilova ${ }^{1,2,5}$, Valsamo Anagnostou 1,2, Ada J. Tam, ${ }^{1,2}$, Brandon S. Luber ${ }^{1,5}$, Bjarne R. Bartlett 1,6,7,10, Laveet K. Aulakh 1,6,7, John-William Sidhom ${ }^{1,2}$, Qingfeng Zhu ${ }^{1,2,3}$, Cynthia L. Sears ${ }^{1,2}$, Leslie Cope ${ }^{1,2,5}$, William H. Sharfman², Elizabeth D. Thompson 1,2,6, Joanne Riemer ${ }^{1,2}$, Kristen A. Marrone ${ }^{1,2}$, Jarushka Naidoo ${ }^{1,2}$, Victor E. Velculescu ${ }^{1,2}$, Patrick M. Forde ${ }^{1,2}$, Bert Vogelstein ${ }^{1,7}$, Kenneth W. Kinzler ${ }^{1,7}$, Nickolas Papadopoulos, ${ }^{1,7}$, Jennifer N. Durham ${ }^{1,2}$, Hao Wang ${ }^{1,2,5}$, Dung T. Le ${ }^{1,2}$, Sune Justesen ${ }^{8}$, Janis M. Taube ${ }^{1,2,3}$, Luis A. Diaz Jr ${ }^{1,6,7,9}$, Julie R. Brahmer ${ }^{1,2}$, Drew M. Pardoll ${ }^{1,2}$, Robert A. Anders ${ }^{1,2,3}$ and Franck Housseau ${ }^{1,2^{*}}$ (D)

\begin{abstract}
Background: Several predictive biomarkers are currently approved or are under investigation for the selection of patients for checkpoint blockade. Tumor PD-L1 expression is used for stratification of non-small cell lung (NSCLC) patients, with tumor mutational burden (TMB) also being explored with promising results, and mismatch-repair deficiency is approved for tumor site-agnostic disease. While tumors with high PD-L1 expression, high TMB, or mismatch repair deficiency respond well to checkpoint blockade, tumors with lower PD-L1 expression, lower mutational burdens, or mismatch repair proficiency respond much less frequently.

Case presentation: We studied two patients with unexpected responses to checkpoint blockade monotherapy: a patient with PD-L1-negative and low mutational burden NSCLC and one with mismatch repair proficient colorectal cancer (CRC), both of whom lack the biomarkers associated with response to checkpoint blockade, yet achieved durable clinical benefit. Both maintained T-cell responses in peripheral blood to oncogenic driver mutations - BRAF-N581I in the NSCLC and AKT1-E17K in the CRC - years after treatment initiation. Mutation-specific T cells were also found in the primary tumor and underwent dynamic perturbations in the periphery upon treatment.

Conclusions: These findings suggest that $T$ cell responses to oncogenic driver mutations may be more prevalent than previously appreciated and could be harnessed in immunotherapeutic treatment, particularly for patients who lack the traditional biomarkers associated with response. Comprehensive studies are warranted to further delineate additional predictive biomarkers and populations of patients who may benefit from checkpoint blockade.
\end{abstract}

Keywords: Checkpoint blockade, Predictive biomarkers, Oncogene, Neoantigens, T cells

\footnotetext{
* Correspondence: fhousse1@jhmi.edu

${ }^{\dagger}$ Kellie N. Smith and Nicolas J. Llosa contributed equally to this work.

${ }^{1}$ Bloomberg-Kimmel Institute for Cancer Immunotherapy, Johns Hopkins

University, Baltimore, MD, USA

${ }^{2}$ Sidney Kimmel Comprehensive Cancer Center, Johns Hopkins University,

Baltimore, MD, USA

Full list of author information is available at the end of the article
}

(c) The Author(s). 2019 Open Access This article is distributed under the terms of the Creative Commons Attribution 4.0 International License (http://creativecommons.org/licenses/by/4.0/), which permits unrestricted use, distribution, and

reproduction in any medium, provided you give appropriate credit to the original author(s) and the source, provide a link to the Creative Commons license, and indicate if changes were made. The Creative Commons Public Domain Dedication waiver (http://creativecommons.org/publicdomain/zero/1.0/) applies to the data made available in this article, unless otherwise stated. 


\section{Background}

Expression of the ligand for PD-1, PD-L1, by tumor cells as well as detection of intratumoral microsatellite instability (MSI-H) were approved as inclusion criteria for anti-PD1 treatment of cancer patients. Clinical response to $\mathrm{PD}-1$ blockade is much more frequent in patients whose tumors have a very high nonsynonymous tumor mutational burden (TMB) and consequent neoantigen expression [1-3], likely indicating the role of $\mathrm{T}$-cells specific for mutation associated neoantigens in facilitating tumor regression. For example, mismatch repair deficient colorectal cancers (MMRd CRC, also MSI-H) which typically have $>1000$ mutations per exome, have an inflamed tumor microenvironment and respond well to $\mathrm{PD}-1$ pathway blockade. In contrast, metastatic mismatch repair proficient CRC (MMRp mCRC, also called microsatellite stable, MSS), which are characterized by a much lower mutational burden and an uninflamed tumor microenvironment [4], rarely respond to PD-1 pathway blockade [1]. Similarly, in non-small cell lung cancer (NSCLC), high TMB cancers respond to checkpoint blockade at significantly higher frequency than those with low $\mathrm{TMB}[2,3]$. However, some patients without MMRd or high TMB cancers derive clinical benefit from PD-1 pathway blockade; understanding the basis for these outlier responses will provide potential biomarkers for therapeutic guidance and may provide insights into improving immunotherapy outcomes in patients whose cancers lack these biomarkers.

Here we report two cases of patients with biomarker-negative tumors: a NSCLC patient whose tumor had 30 mutations and was negative for PD-L1 and a MMRp CRC patient, both of whom derived durable clinical benefit from PD-1 blockade monotherapy. Each patient maintained a T-cell response to a hotspot oncogenic mutation many years after treatment initiation: BRAF N581I $[5,6]$ in the NSCLC patient and AKT1 E17K $[7,8]$ in the CRC patient. These findings suggest that neoantigens derived from oncogenic driver mutations induce endogenous T-cell responses, which may be particularly efficacious in lower mutational burden tumors owing to the likelihood that oncogenic mutations are required for tumor survival.

\section{Case presentations}

We evaluated two patients with tumors anticipated to be non-responsive to immunotherapy who derived durable clinical benefit and prolonged overall survival from anti-PD-1 therapy - both remain alive with ECOG-0 performance status years later. The first patient, LUAD-3001, is a 76-year-old woman who underwent a right lower lung lobectomy in 2012 for a T3 N0 well-to-moderately differentiated mucinous adenocarcinoma. Nine months after finishing adjuvant chemotherapy, three new lung nodules were found on surveillance imaging. Biopsy confirmed recurrent adenocarcinoma. The patient was enrolled on a clinical trial of single-agent nivolumab in December 2013 (NCT01454102), with therapy ongoing through the present. By July 2014, the metastases had completely disappeared, and complete response continues to be maintained for 4.5 years. Figure 1 a shows LUAD3001 CT before treatment, at first follow up, as well as 2.5 and 4 years after follow up. Whole exome sequencing revealed the patient's tumor had 30 nonsynonymous exome mutations and was negative for ALK, EGFR, ROS1, and KRAS abnormalities. The tumor harbored an oncogenic BRAF N581I mutation [5, 6] (Additional file 1: Table S1). The tumor cells were negative for PD-L1 expression (Fig. 1b, center), though prominent perivascular lymphoid aggregates were PD-L1+. Immunophenotyping confirmed the presence of $\mathrm{CD}^{+} \mathrm{T}$ lymphocytes (Fig. 1b, right).

The second patient, CRC-010, is a 69 year old woman initially diagnosed with a stage III mucinous right-sided colon adenocarcinoma. PD-L1 expression in her original primary tumor was observed at the interface of tumor and normal tissue and there was a dense $\mathrm{CD} 8^{+}$lymphocytic infiltrate (Fig. 1d). Staining for mismatch repair enzymes was normal, consistent with a mismatch repair proficient genotype. Whole exome sequencing of the primary lesion revealed 118 mutations, including oncogenic BRAF V600E and AKT1-E17K mutations. There were no mutations in any of the genes encoding mismatch repair proteins, KRAS or NRAS (Additional file 1: Table S1Table S2).Ten years after a right hemicolectomy, FOLFOX adjuvant therapy and FOLFIRI/cetuximab, she developed a pancreatic metastatic recurrence in 2013. The patient began pembrolizumab therapy in January 2014. CT scans in April 2014 showed disease stabilization. In May 2014, she discontinued pembrolizumab after 4 doses due to grade 3 serum pancreatic enzyme elevation. CT at the time showed stable disease with no new metastases (Fig. 1c). Following a course of chemoradiation with capecitabine, and then FOLFOX/Bevacizumab, she has not received further therapy since May 2015 and imaging studies continue to show a stable pancreatic mass with no new lesions (Fig. 1c). A fine needle aspiration biopsy of the pancreatic mass performed at the end of treatment in June 2015 demonstrated the presence of clusters of neoplastic cells compatible with residual moderately differentiated adenocarcinoma with mucinous features and a brisk infiltrate of $\mathrm{CD} 8^{+}$T-cells (Fig. 1d). 
$\mathbf{a}$
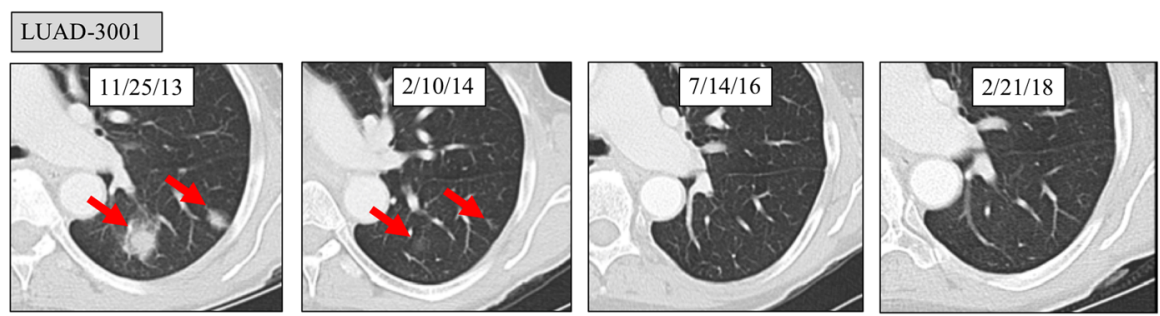

b
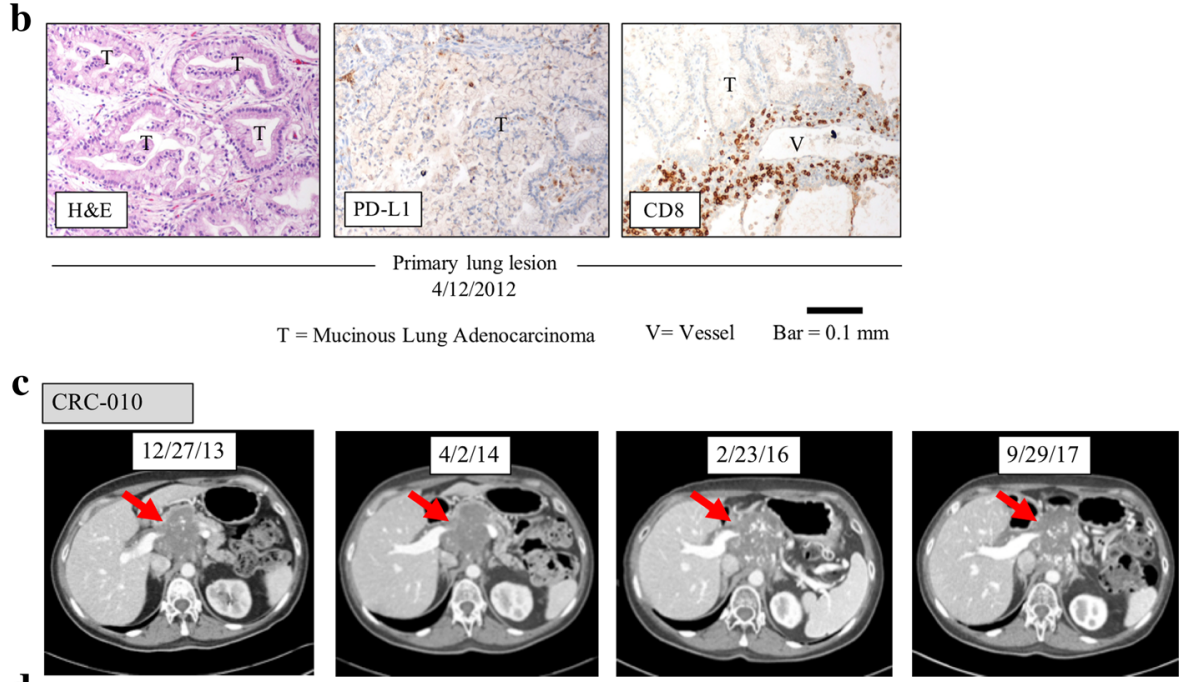

d
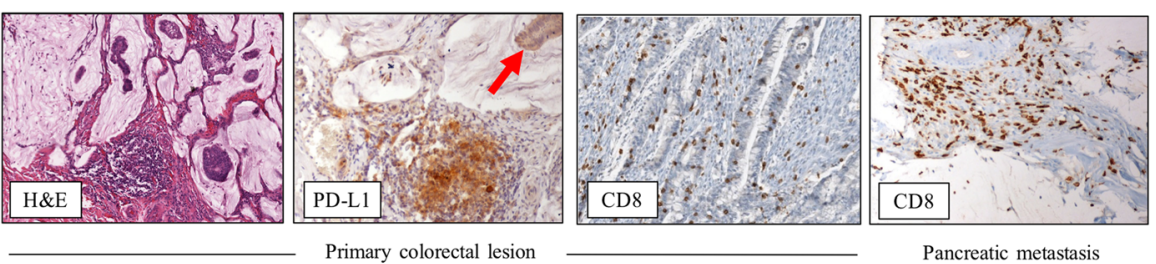

9/29/2003

ancreatic metastasis
$12 / 30 / 2013$

Fig. 1 Durable clinical benefit to PD-1 blockade in two patients without high mutational burden tumors. a, Patient LUAD-3001 - a 76 year old woman with metastatic non-small cell lung cancer. Selected cropped IV contrast enhanced $C T$ images of the chest in lung window at four different timepoints. Baseline exam (11/25/13) demonstrates two left lower lobe solid nodules with surrounding ground glass opacities (red arrows) compatible with metastases. First followup exam while on nivolumab (2/10/14) demonstrates near complete resolution with minimal residual ground glass opacities (red arrows). Additional two and four year followup exams (7/14/16 and 2/21/18) demonstrate complete and durable resolution of metastases, with no evidence of progression elsewhere in the body (not shown). b, H\&E staining (left panel), PD-L1 staining (center panel), and CD8 infiltration (right panel) of the primary tumor obtained from patient LUAD-3001 during surgical resection on 4/12/2012. c, Patient CRC-010 - a 69 year old woman with metastatic recurrent mismatch repair proficient colorectal cancer with locally invasive pancreatic metastasis. Selected IV contrast enhanced $C T$ images of the abdomen in venous phase. Baseline exam (12/27/13) demonstrates a heterogeneous hypovascular mass with scattered calcifications (red arrow). Four month follow up exam on pembrolizumab (4/2/14) demonstrates slight enlargement without new metastases. Metastasis slowly decreased in size on 2 year follow up (2/23/16) and slightly increased on four year follow up exam (9/29/17). No new metastases are seen on interval or latest CT exam and disease remains stable. d, H\&E staining (left panel), PD-L1 staining showing no expression on tumor cells (red arrow, center left panel) but high expression at the invasive front on a discrete immune cell aggregate and CD8 infiltration (center right panel) in the primary tumor obtained from patient CRC-010 during surgical resection on 9/29/2003. CD8 staining demonstrating a brisk CD8 ${ }^{+}$lymphocytic infiltrate is also shown on a fine needle aspiration of the pancreatic recurrence on 12/30/2013 (right panel)

\section{Methods}

\section{Patient selection and tumor samples}

The patients described in this study provided informed consent as approved by the IRB of Johns Hopkins. Patient LUAD-3001 was enrolled to CheckMate 012, a phase I study evaluating nivolumab combination therapy in subjects with stage IIIb/IV non-small cell lung cancer (NSCLC; clinicaltrials.gov, NCT01454102) and was treated with nivolumab monotherapy [9]. Patient CRC-010 was enrolled to a phase II study of pembrolizumab treatment for metastatic colorectal cancer (CRC; clinicaltrials.gov, NCT018706511) [1]. The samples used for each analysis in this study are detailed in Additional file 1: Table S1Table S3. 


\section{Histopathology, immunohistochemistry and image analysis}

Tissue specimens were stained with hematoxylin and eosin combination (H\&E). Formalin-fixed paraffin embedded (FFPE) tissue sections were stained for CD8 (clone C8144B, Cell Marque, Rocklin, CA) and PD-L1 (clone E1L3N) as previously reported [10].

\section{Whole exome sequencing (WES), neoantigen prediction, and in vitro peptide binding assays}

Tumor and normal WES were compared to identify somatic alterations using the VariantDx software pipeline [11]. Mutations from WES combined with each patient's major histocompatibility complex class I haplotype were applied in the ImmunoSelect- $\mathrm{R}$ neoantigen prediction platform (Personal Genome Diagnostics) [11]. This algorithm predicts the MHC class I binding potential of each somatic and wild-type peptide. Neoantigen candidates were further filtered by tumor-associated expression levels derived from TCGA to generate a final peptide ranking for experimental testing. Lollipop plots showing mutations detected in the $B R A F$ and $A K T 1$ genes were generated by cBioPortal $[12,13]$. Binding assays were performed as previously described [14].

\section{Peripheral blood T-cell reactivity and bioinformatic identification of mutation associated neoantigen-specific T-cell clonotypes}

We used the MANAFEST (Mutation Associated NeoAntigen Functional Expansion of Specific T-cells) assay [15] to evaluate $\mathrm{T}$-cell responsiveness to mutation-associated neoantigens. Briefly, putative neoantigenic peptides defined by the ImmunoSelect- $\mathrm{R}$ pipeline (see above [11]; Additional file 1: Table S1Tables S4 and S5) were synthesized (Sigma-Aldrich) and used to stimulate T-cells in vitro for 10 days as previously described [15]. T-cell receptor sequencing (TCRseq; Adaptive Biotechnologies) [16] was performed on individual peptide-stimulated T-cell cultures and T-cells cultured without peptide. Bioinformatic analysis of productive clones was performed to identify antigen-specific T-cell clonotypes meeting the following criteria: 1) significant expansion (Fisher's exact test with Benjamini-Hochberg correction for FDR, $p<0.0001$ ) compared to T-cells cultured without peptide, 2) significant expansion compared to every other peptide-stimulated culture $(\mathrm{FDR}<0.0001), 3)$ an odds ratio $>5$ compared to the "no peptide" control, 4) a minimum of 10 templates detected by TCRseq, and 5) reached a minimum baseline threshold to ensure adequate distribution among culture wells or was detected in a repeat stimulation experiment. TCRseq was also performed on DNA extracted from tumor tissue obtained from the primary surgical resection and serial peripheral blood samples where available. TCRseq was performed using the survey resolution ImmunoSEQ ${ }^{\circ}$ platform for tissue and MANAFEST samples and deep resolution sequencing for peripheral blood samples [16].

\section{Results}

T-cell recognition of mutation-associated neoantigens

To determine if patients LUAD-3001 and CRC-010 had circulating $\mathrm{T}$-cell clones that recognized tumor neoantigens and that were also present in the tumor, we used the MANAFEST assay [15], in which short-term cultures of peripheral blood T-cells with individual candidate mutation associated neoantigen peptides predicted by an HLA-I allele-specific algorithm are analyzed via $\mathrm{T}$-cell receptor sequencing (TCRseq) [16]. Twenty three out of 26 candidate neoantigenic peptides tested induced significant and specific clonotypic expansions of $\mathrm{CD}^{+} \mathrm{T}$ cells obtained from patient LUAD-3001 2 years after anti-PD-1 treatment initiation (Additional file 1: Table S1Table S6). Two of these neoantigens (a 10mer and an 11mer; LUAD 26 and LUAD 31) contained the oncogenic driver mutation BRAF N581I, a hotspot mutation previously reported to recurrently occur in melanoma and colorectal cancer [5, 6, 17]. The oncogenic mechanism underlying BRAF N581I is distinct from that of BRAF V600E in that N581I has diminished or inactive BRAF kinase activity but induces KRAS-dependent CRAF signaling and ERK activation [17]. Three $\mathrm{T}$-cell clones reactive with $\mathrm{BRAF}$ N581I recognized LUAD 26, a HLA-A*02:01-restricted epitope (IIFLHEDLTV; Fig. 2a, Additional file 1: Table S1Table S6); one of these clones was detected in the original primary tumor resection. All three of these clones were detected in peripheral blood T cells obtained prior to treatment, and were present at much lower frequency by 12 weeks after treatment initiation and after complete tumor regression. Although having seemingly poor binding affinity for HLA-A*02:01, LUAD 26 demonstrated improved binding kinetics relative to its wild-type counterpart (Fig. 2c). T cell recognition of hotspot BRAF mutations have previously been described $[18,19]$, but this is the first report of a $\mathrm{T}$ cell response against neoantigens derived from mutations at position 581. Remarkably, T-cell recognition of the BRAF N581I oncogenic driver and 22 additional mutation-associated neoantigens described herein (Additional file 1: Table S1Table S6) persisted years after complete tumor regression, thereby defining a preexisting and long-lived antitumor memory T-cell response.

Similarly to patient LUAD-3001, we detected T cell reactivity to the oncogenic driver mutation, AKT1 E17K, in peripheral $\mathrm{T}$ cells obtained 3 years post-anti-PD-1 from patient CRC-010, a patient with MMRp mCRC. We identified two specifically recognized mutation-associated neoantigen peptides (Additional file 1: Table S1Table S6), 


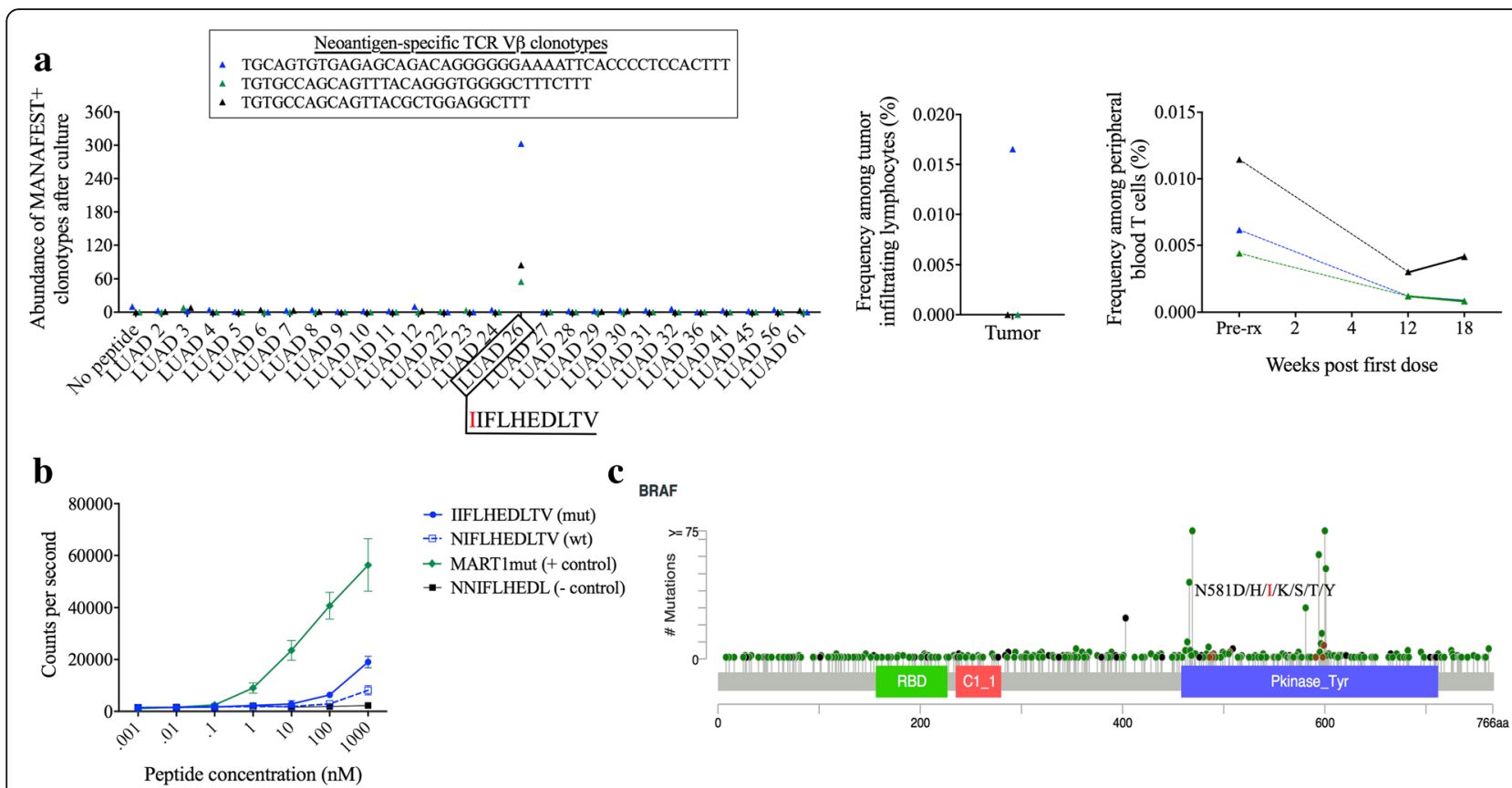

Fig. 2 T-cell recognition of BRAF N5811 mutation in lung cancer patient LUAD-3001 responding to anti-PD-1 treatment. Individual 10-day peptide-stimulated cultures identified persistent mutation associated neoantigen-specific clonotypes (described in methods) detectable in the blood of patient LUAD-3001 > 2 years after complete tumor regression following PD-1 blockade. a Three clonotypes recognized the A*02:01-restricted BRAF N5811-derived IIFLHEDLTV peptide neoantigen (LUAD 26, left panel). The TGCAGTGTGAGAGCAGACAGGGGGGAAAATTCACCCCTCCACTTT clonotype was detected in the original resected tumor (center panel), whereas all three clonotypes were detected in serial peripheral blood samples obtained before and after PD-1 blockade (right panel). Data are shown as the number of cells detected after the 10 day culture (abundance) for cultured cells and the relative frequency (\%) of each clonotype among all cells detected by TCRseq for FFPE tumor tissue and serial peripheral blood samples. b Duplicate binding assays were performed on the putative neoantigen and wild type counterpart, as well as the known MART1 mutant HLA A*02:01-restricted ELAGIGILTV epitope. Data are shown as mean counts per second, with error bars representing the standard deviation. c The lollipop plot shows the position of the patient's BRAF N581I mutation among the other oncogenic mutations within the BRAF gene; green: missense mutations, black: truncating mutations, brown: inframe mutations, purple: other

including an AKT1 E17K - derived, HLA-A*23:01-restricted KYIKTWRPRYF peptide epitope (CRC 8) that induced a single expanded TCRV $\beta$ clonotype (Fig. 3a). This clone persisted in the periphery of the tumor-bearing patient as evidenced by its detection in a a subsequent blood sample collected one year later (data not shown). Strikingly, this single T-cell clone comprised $1.4 \%$ of tumor infiltrating lymphocytes detected in the original primary colon tumor of patient CRC-010 and underwent rapid expansion in the periphery upon PD-1 blockade before returning to pre-treatment frequency by 20 weeks post-treatment (Fig. 3a). This neoantigen demonstrated high affinity binding to $A^{*} 23: 01$ in an in vitro assay, with similar binding kinetics observed in the wild-type peptide (Fig. 3b). The E17K mutation is a "hotspot" in AKT1, accounting for the majority of mutations causing constitutive activation of the kinase [8].

\section{Discussion and conclusions}

These findings demonstrate that driver mutations may elicite efficient long-lived endogenous anti-tumor immune responses, and these responses may facilitate clinical response in patients treated with checkpoint blockade. On this note, adoptive transfer of $\mathrm{T}$ cells specific for hotspot driver oncogenic mutations, including $\mathrm{CD}^{+} \mathrm{T}$ cells specific for an HLA class I-restricted KRAS G12D epitope [20] and CD4 ${ }^{+} \mathrm{T}$ cells specific for an HLA class II-restricted BRAF V599E [19] or BRAF V600E [18] mutation have proven to derive clinical benefit. Here we provide further demonstration that endogenous memory $\mathrm{T}$-cells targeting such oncogenic driver mutations can persist in the peripheral blood for many years after tumor clearance. Interestingly, the BRAF N581I-derived neoantigen demonstrated limited affinity for HLA-A*02:01 in our in vitro binding assay (Fig. 2b). Lower affinity epitopes $(>500 \mathrm{nM})$ are not uncommon [21-23] and in some instances binding to HLA might be subsequently enhanced by post-translational modifications [24]. This is in contrast to the high affinity of the AKT1 E17K-derived neoantigen and its wild-type counterpart. In this case,the mutated amino acid is located at position 1 and therefore unlikely to affect binding to the MHC but may interfere with cognate TCR binding.

The identification of immunogenic oncogene-derived neoantigens has profound clinical implications. In contrast 


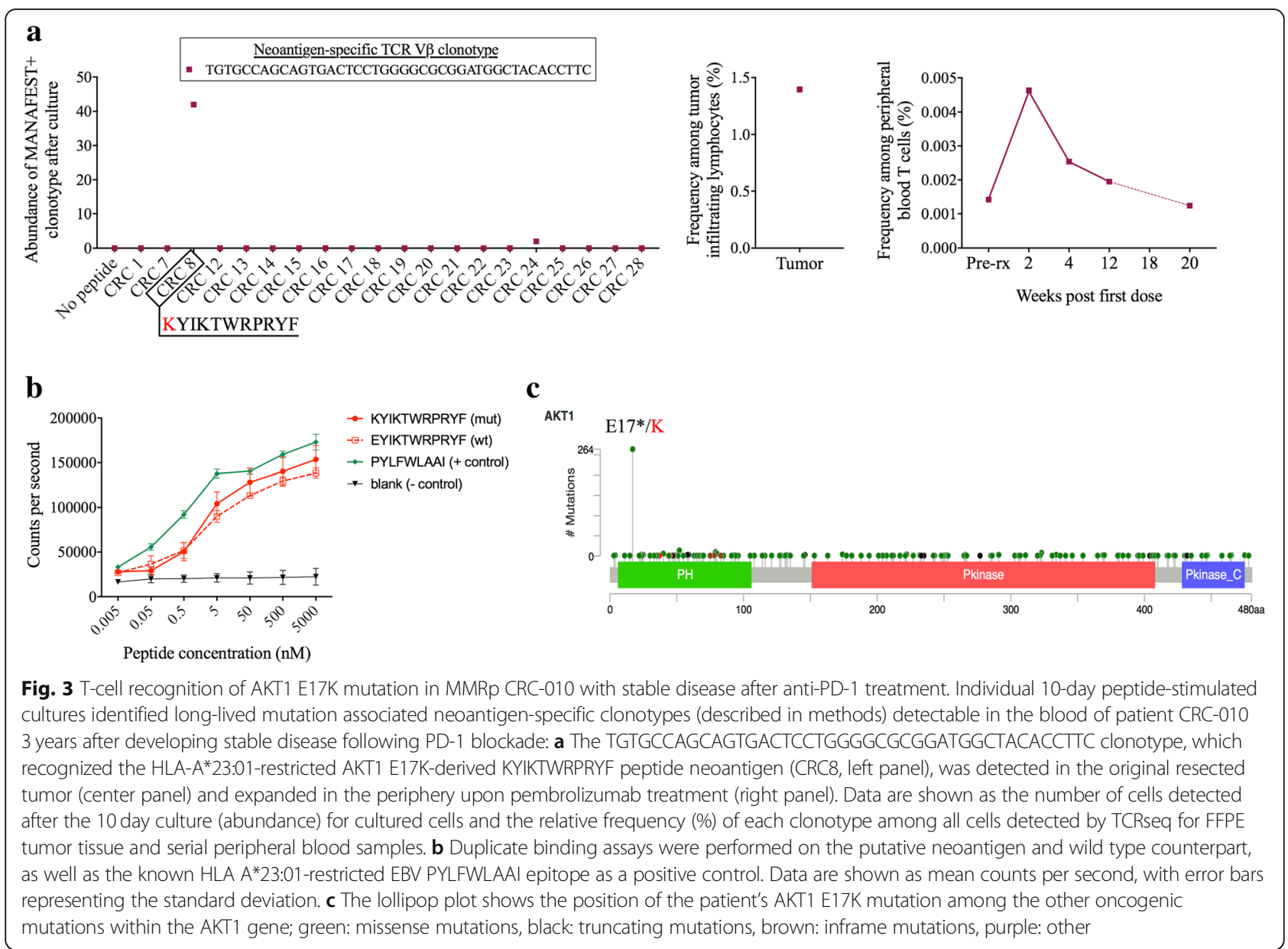

to passenger mutations, driver oncogenic mutations are less likely to be eliminated by the tumor as a means of immune escape, since they are required for the transformed phenotype. Thus, sustained $\mathrm{T}$ cell responses against driver mutations likely are more productive for long term tumor control [25]. New bioassays to detect and monitor the immune response to neoantigens, such as the MANAFEST assay used here, will help systematic screening for T-cell responses against tumor-specific mutations with a special emphasis on oncogenic driver mutations. In addition, the development of new assays that enable concurrent phenotypic profiling of neoantigen-specific $\mathrm{T}$ cell clonotypes will shed light on the effector function of these $\mathrm{T}$ cells. They may delineate a patient population that would not otherwise be predicted to respond to checkpoint blockade based on current biomarkers, such as tumor PD-L1 expression, high TMB, or mismatch repair status. While these findings do not provide evidence that the oncogene reactive $T$ cells facilitated durable clinical benefit in these patients, they provide the foundation for further exploration of biomarkers likely to identify previously unappreciated populations of patients eligible for checkpoint blockade-based clinical trials. Indeed, patient LUAD-
3001 would not currently be eligible to receive anti-PD-1 monotherapy as standard of care in the first line setting, as she did as part of CHECKMATE 012, and patient CRC-010 would not be eligible to receive anti-PD-1 at all. Additionally, identifying $\mathrm{T}$ cell clonotypes specific for these mutations provides the foundation for vaccines or $\mathrm{T}$ cell therapies targeting oncogene mutation-derived neoantigens in patients who did not mount these responses endogenously. High TMB, tumor PD-L1 expression, and MMR status being imperfect predictive biomarkers, new sensitive next generation sequencing methods, T-cell detection assays, and epitope prediction algorithms allow for the systematic screening of cancer patients for reactivity towards shared driver mutations, the detection of which could provide additional predictive value for clinical benefit to checkpoint blockade.

\section{Additional file}

Additional file 1: Table S1. Sequence alterations detected in the primary tumor of LUAD-3001, Table S2. Sequence alterations detected in the primary tumor of CRC-010, Table S3. Specimen characteristics used for genomic and immunologic analyses, Table S4. Putative neoantigens 
tested in LUAD-3001, Table S5. Putative neoantigens tested in CRC-010 Table S6. Neoantigen-specific T cell clonotypes detected in patients LUAD-3001 and CRC-010 (XLSX 98 kb)

\section{Abbreviations}

NSCLC: Non-small cell lung cancer; CRC: Colorectal cancer; PD1: Programmed death 1; PD-L1: Programmed death ligand 1; TMB: Tumor mutational burden; MMRd: Mismatch repair deficient; MSI-H: Microsatellite instabibility high; MMRp: Mismatch repair proficient; MSS: Microsatellite stable; ECOG: Eastern Cooperative Oncology Group; FFPE: Formalin fixed, paraffin embedded; CD8: Cluster of differentiation 8; WES: Whole exome sequencing; TCGA: The Cancer Genome Atlas; MANAFEST: Mutation associated neoantigen functional expansion of specific T cells; TCRseq: $T$ cell receptor sequencing; DNA: Deoxyribonucleic acid; HLA: Human leukocyte antigen; TCRV $\beta$ : T cell receptor variable gene, $\beta$ chain; MHC: Major histocompatibility complex

\section{Acknowledgements}

We would like to thank the patients and their families, as well as Lexa Hartman and other members of our respective clinical and laboratory research teams for their assistance with these studies.

\section{Funding}

This work was supported by Bloomberg Philanthropies, the Swim Across America Laboratory at Johns Hopkins, the Lung Cancer Foundation of America and the International Association for the Study of Lung Cancer Foundation, LUNGevity, The V Foundation, The Mark Foundation for Cancer Research, NIH Gastrointestinal Specialized Programs of Research Excellence grant (SPORE; P50 CA062924) and NIH grants P30 DK089502, P30 CA006973, R01 CA203891, R01 CA121113, U10 CA180950, and T32 CA193145, the Commonwealth foundation, Cancer Research Institute/FightColorectalCancer Merck, Bristol Myers Squibbb, a Stand Up to Cancer Immunology Dream Team Translational Research Grant (SU2C-AACR-DT1012), and the Colorectal Cancer Dream Team Translational Research Grant (SU2C-AACR-DT22-17). Stand Up to Cancer is a program of the Entertainment Industry Foundation. Research grants are administered by the American Association for Cancer Research, the scientific partner of SU2C.

\section{Availability of data and materials}

The datasets used and/or analysed during the current study are available from the corresponding author on reasonable request.

\section{Authors' contributions}

KNS, NJL, FH, and RAA designed and executed all experiments and led data analysis and manuscript preparation. TRC, ET, RAA, and JMT performed histopathological assessments. FV evaluated radiographic responses. BV, KWK $N P, V A, W$, and LAD performed and analyzed whole exome sequencing data. JR, KAM, PMF, JN, DTL, LAD, and JRB oversaw patient care and treatment. LD, AJT, BSL, WS, QZ, and HW perform data analysis and statistical oversight. NS, HF, PS, HYC, HG, TO, AHA, AJT, BRB, LKA, and JS provided technical assistance. SJ performed peptide binding and stability assays. Al authors contributed to data analysis and manuscript preparation. All authors read and approved the final manuscript

\section{Ethics approval and consent to participate}

The patients described in this study provided written informed consent as approved by the IRB of Johns Hopkins.

\section{Consent for publication}

Not applicable.

\section{Competing interests}

K.N.S., F.H., D.M.P, V.A., B.V., K.W.K., N.P., L.A.D, and V.E.V. have filed for patent protection on a subset of the technologies described herein (US provisional application no. 62/407,820). D.M.P. has ownership interest (including patents) in BMS, Medlmmune/AstraZeneca, and Potenza, and is a consultant/advisory board member for BMS and Medlmmune/AstraZeneca. V.E.V. has ownership interest (including patents) in Personal Genome Diagnostics and is a consultant/advisory board member for the same.

\section{Publisher's Note}

Springer Nature remains neutral with regard to jurisdictional claims in published maps and institutional affiliations.

\section{Author details}

${ }^{1}$ Bloomberg-Kimmel Institute for Cancer Immunotherapy, Johns Hopkins University, Baltimore, MD, USA. ${ }^{2}$ Sidney Kimmel Comprehensive Cancer Center, Johns Hopkins University, Baltimore, MD, USA. ${ }^{3}$ Department of Pathology, Johns Hopkins University, Baltimore, MD, USA. ${ }^{4}$ Russell H. Morgan Department of Radiology and Radiological Science, Johns Hopkins University, Baltimore, MD, USA. ${ }^{5}$ Division of Biostatistics and Bioinformatics, Johns Hopkins University, Baltimore, MD, USA. ${ }^{6}$ The Swim Across America Laboratory, John Hopkins University, Baltimore, MD, USA. 'Ludwig Center and Howard Hughes Medical Institute, Johns Hopkins University, Baltimore, MD, USA. ${ }^{8}$ Immunitrack, Copenhagen, Denmark. ${ }^{9}$ Department of Medicine, Division of Solid Tumor Oncology, Memorial Sloan Kettering Cancer Center, New York, NY, USA. ${ }^{10}$ Present address: B.R.B.,Bioinformatics Core, Department of Complementary \& Integrative Medicine, University of Hawaii John A. Burns School of Medicine, Honolulu, HI 96813, USA.

Received: 14 September 2018 Accepted: 20 December 2018 Published online: 11 February 2019

\section{References}

1. Le DT, Uram JN, Wang H, Bartlett BR, Kemberling H, Eyring AD, et al. PD-1 Blockade in Tumors with Mismatch-Repair Deficiency. N Engl J Med. 2015; 372:2509-20.

2. Rizvi NA, Hellmann MD, Snyder A, Kvistborg P, Makarov V, Havel JJ, et al. Cancer immunology. Mutational landscape determines sensitivity to PD-1 blockade in non-small cell lung cancer. Science. 2015;348:124-8.

3. Hellmann MD, Ciuleanu TE, Pluzanski A, Lee JS, Otterson GA, AudigierValette C, et al. Nivolumab plus Ipilimumab in Lung Cancer with a High Tumor Mutational Burden. N Engl J Med. 2018;378:2093-104.

4. Llosa NJ, Cruise M, Tam A, Wicks EC, Hechenbleikner EM, Taube JM, et al. The vigorous immune microenvironment of microsatellite instable colon cancer is balanced by multiple counter-inhibitory checkpoints. Cancer Discov. 2015:5:43-51.

5. Wong CW, Fan YS, Chan TL, Chan AS, Ho LC, Ma TK, et al. BRAF and NRAS mutations are uncommon in melanomas arising in diverse internal organs. $J$ Clin Pathol. 2005;58:640-4.

6. Carter J, Tseng LH, Zheng G, Dudley J, Illei P, Gocke CD, et al. Non-p.V600E BRAF Mutations Are Common Using a More Sensitive and Broad Detection Tool. Am J Clin Pathol. 2015;144:620-8.

7. Hechtman JF, Sadowska J, Huse JT, Borsu L, Yaeger R, Shia J, et al. AKT1 E17K in Colorectal Carcinoma Is Associated with BRAF V600E but Not MSI-H Status: A Clinicopathologic Comparison to PIK3CA Helical and Kinase Domain Mutants. Mol Cancer Res. 2015;13:1003-8.

8. Carpten JD, Faber AL, Horn C, Donoho GP, Briggs SL, Robbins CM, et al. A transforming mutation in the pleckstrin homology domain of AKT1 in cancer. Nature. 2007:448:439-44.

9. Hellmann MD, Rizvi NA, Goldman JW, Gettinger SN, Borghaei H, Brahmer JR, et al. Nivolumab plus ipilimumab as first-line treatment for advanced non-small-cell lung cancer (CheckMate 012): results of an open-label, phase 1. multicohort study. Lancet Oncol. 2017:18:31-41.

10. Taube JM, Klein A, Brahmer JR, Xu H, Pan X, Kim JH, et al. Association of PD-1, PD-1 Ligands, and Other Features of the Tumor Immune Microenvironment with Response to Anti-PD-1 Therapy. Clin Cancer Res. 2014;20:5064-74.

11. Jones S, Anagnostou V, Lytle K, Parpart-Li S, Nesselbush M, Riley DR, et al. Personalized genomic analyses for cancer mutation discovery and interpretation. Sci Transl Med. 2015;7:283ra53.

12. Cerami E, Gao J, Dogrusoz U, Gross BE, Sumer SO, Aksoy BA, et al. The cBio cancer genomics portal: an open platform for exploring multidimensional cancer genomics data. Cancer Discov. 2012;2:401-4.

13. Gao J, Aksoy BA, Dogrusoz U, Dresdner G, Gross B, Sumer SO, et al. Integrative analysis of complex cancer genomics and clinical profiles using the cBioPortal. Sci Signal. 2013;6:pl1.

14. Le DT, Durham JN, Smith KN, Wang H, Bartlett BR, Aulakh LK, et al. Mismatch repair deficiency predicts response of solid tumors to PD-1 blockade. Science. 2017;357:409-13. 
15. Danilova L, Anagnostou V, Caushi JX, Sidhom JW, Guo H, Chan HY, et al The Mutation-Associated Neoantigen Functional Expansion of Specific T Cells (MANAFEST) Assay: A Sensitive Platform for Monitoring Antitumor Immunity. Cancer Immunol Res. 2018;6:888-99.

16. Carlson CS, Emerson RO, Sherwood AM, Desmarais C, Chung MW, Parsons $J M$, et al. Using synthetic templates to design an unbiased multiplex PCR assay. Nat Commun. 2013;4:2680

17. Yao Z, Yaeger R, Rodrik-Outmezguine VS, Tao A, Torres NM, Chang MT, et al. Tumours with class 3 BRAF mutants are sensitive to the inhibition of activated RAS. Nature. 2017;548:234-8.

18. Veatch JR, Lee SM, Fitzgibbon M, Chow IT, Jesernig B, Schmitt T, et al. Tumor-infiltrating BRAFV600E-specific CD4+ T cells correlated with complete clinical response in melanoma. J Clin Invest. 2018;128:1563-8.

19. Sharkey MS, Lizee G, Gonzales MI, Patel S. Topalian SL. CD4(+) T-cell recognition of mutated B-RAF in melanoma patients harboring the V599E mutation. Cancer Res. 2004;64:1595-9.

20. Tran E, Robbins PF, Lu YC, Prickett TD, Gartner JJ, Jia L, et al. T-Cell Transfer Therapy Targeting Mutant KRAS in Cancer. N Engl J Med. 2016;375:2255-62.

21. Assarsson E, Sidney J, Oseroff C, Pasquetto V, Bui HH, Frahm N, et al. A quantitative analysis of the variables affecting the repertoire of $\mathrm{T}$ cell specificities recognized after vaccinia virus infection. J Immunol. 2007;178: 7890-901.

22. Paul S, Weiskopf D, Angelo MA, Sidney J, Peters B, Sette A. HLA class I alleles are associated with peptide-binding repertoires of different size, affinity, and immunogenicity. J Immunol. 2013;191:5831-9.

23. Sette A, Vitiello A, Reherman B, Fowler P, Nayersina R, Kast WM, et al. The relationship between class I binding affinity and immunogenicity of potential cytotoxic T cell epitopes. J Immunol. 1994;153:5586-92.

24. Sidney J, Vela JL, Friedrich D, Kolla R, von Herrath M, Wesley JD, et al. Low HLA binding of diabetes-associated CD8+ T-cell epitopes is increased by post translational modifications. BMC Immunol. 2018;19:12.

25. Dudnik E, Peled N, Nechushtan H, Wollner M, Onn A, Agbarya A, et al. BRAF Mutant Lung Cancer: Programmed Death Ligand 1 Expression, Tumor Mutational Burden, Microsatellite Instability Status, and Response to Immune Check-Point Inhibitors. J Thorac Oncol. 2018;13:1128-37.

Ready to submit your research? Choose BMC and benefit from:

- fast, convenient online submission

- thorough peer review by experienced researchers in your field

- rapid publication on acceptance

- support for research data, including large and complex data types

- gold Open Access which fosters wider collaboration and increased citations

- maximum visibility for your research: over $100 \mathrm{M}$ website views per year

At $\mathrm{BMC}$, research is always in progress.

Learn more biomedcentral.com/submissions 


\title{
Correction to: persistent mutant oncogene specific $T$ cells in two patients benefitting from anti-PD-1
}

\author{
Kellie N. Smith ${ }^{1,2+}$, Nicolas J. Llosa ${ }^{1,2+}$, Tricia R. Cottrell ${ }^{1,2,3}$, Nicholas Siegel ${ }^{1,2}$, Hongni Fan ${ }^{1,2}$, Prerna Suri ${ }^{1,2}$, \\ Hok Yee Chan 1,2, Haidan Guo ${ }^{1,2}$, Teniola Oke ${ }^{1,2}$, Anas H. Awan 1,2, Franco Verde ${ }^{4}$, Ludmila Danilova 1,2,5, \\ Valsamo Anagnostou ${ }^{1,2}$, Ada J. Tam, ${ }^{1,2}$, Brandon S. Luber ${ }^{1,5}$, Bjarne R. Bartlett 1,6,7,10, Laveet K. Aulakh 1,6,7, \\ John-William Sidhom ${ }^{1,2}$, Qingfeng Zhu ${ }^{1,2,3}$, Cynthia L. Sears ${ }^{1,2}$, Leslie Cope ${ }^{1,2,5}$, William H. Sharfman², \\ Elizabeth D. Thompson 1,2,6, Joanne Riemer ${ }^{1,2}$, Kristen A. Marrone ${ }^{1,2}$, Jarushka Naidoo ${ }^{1,2}$, Victor E. Velculescu ${ }^{1,2}$, \\ Patrick M. Forde ${ }^{1,2}$, Bert Vogelstein ${ }^{1,7}$, Kenneth W. Kinzler ${ }^{1,7}$, Nickolas Papadopoulos ${ }^{1,7}$, Jennifer N. Durham ${ }^{1,2}$, \\ Hao Wang ${ }^{1,2,5}$, Dung T. Le ${ }^{1,2}$, Sune Justesen ${ }^{8}$, Janis M. Taube ${ }^{1,2,3}$, Luis A. Diaz Jr 1,6,7,9, Julie R. Brahmer ${ }^{1,2}$, \\ Drew M. Pardoll ${ }^{1,2}$, Robert A. Anders ${ }^{1,2,3}$ and Franck Housseau ${ }^{1,2^{*}}$
}

\section{Correction to: Journal for ImmunoTherapy of Cancer 2019 7:40 https://doi.org/10.1186/s40425-018-0492-x}

Following publication of the original article [1], it was reported that not all authors' competing interests were stated. The updated Competing Interests can be seen below.

\section{Competing interests}

K.N.S., F.H., D.M.P., V.A., V.E.V., B.V., K.W.K., N.P. and L.A.D. have filed for patent protection on a subset of the technologies described herein (US provisional application no. 62/407,820).

D.M.P. has ownership interest (including patents) in BMS, MedImmune/AstraZeneca, and Potenza, and is a consultant/advisory board member for BMS and MedImmune/AstraZeneca.

V.E.V. is a founder of, holds equity in, and is a member of the Board of Directors of Personal Genome Diagnostics (PGDx). Under license agreements between PGDx, as well other companies, and the Johns Hopkins University, V.E.V. is entitled to a share of royalties received by the University on sales of services or products

\footnotetext{
* Correspondence: fhousse1@jhmi.edu

${ }^{\dagger}$ Kellie N. Smith and Nicolas J. Llosa contributed equally to this work.

${ }^{1}$ Bloomberg-Kimmel Institute for Cancer Immunotherapy, Johns Hopkins University, Baltimore, MD, USA

${ }^{2}$ Sidney Kimmel Comprehensive Cancer Center, Johns Hopkins University, Baltimore, MD, USA

Full list of author information is available at the end of the article
}

described in this paper. Within the last five years, V.E.V. has been an advisor to Daiichi Sankyo, Janssen Diagnostics, Ignyta, and Takeda Pharmaceuticals. These arrangements have been reviewed and approved by the Johns Hopkins University in accordance with its conflict of interest policies.

B.V., K.W.K., \& N.P. are members of the Scientific Advisory Board of Sysmex and are founders of PapGene and Personal Genome Diagnostics and advise Sysmex. KWK \& BV advise Eisai, Phoremost, and Morphotek, and BV is also an advisor to Camden Partners. The companies named above, as well as other companies have licensed previously described technologies related to the work described in this paper from Johns Hopkins University. Some of these licenses are or will be associated with equity or royalty payments to BV, KWK, NP, and IK. Additional patent applications on the work described in this paper may be filed by Johns Hopkins University. The terms of all these arrangements are being managed by Johns Hopkins University in accordance with its conflict of interest policies.

D.T.L. serves on advisory boards for Merck and Bristol Myers Squibb and has received research funding from Merck, Bristol Myers Squibb, Aduro Biotech, Curegenix, and Medivir. She has received speaking honoraria from Merck and is an inventor of licensed intellectual property related to technology for mismatch repair deficiency for diagnosis and therapy (WO2016077553A1) from Johns Hopkins University. 
The terms of these arrangements are being managed by Johns Hopkins.

L.A.D. is a member of the board of directors of Personal Genome Diagnostics (PGDx) and Jounce Therapeutics. LAD holds equity in PapGene, Personal Genome Diagnostics (PGDx) and Phoremost. He is a paid consultant for Merck, PGDx and Phoremost. $\mathrm{LAD}$ is an inventor of licensed intellectual property related to technology for circulating tumor DNA analyses and mismatch repair deficiency for diagnosis and therapy (WO2016077553A1) from Johns Hopkins University. These licenses and relationships are associated with equity or royalty payments to LAD. The terms of all these arrangements are being managed by Johns Hopkins and Memorial Sloan Kettering in accordance with their conflict of interest policies. In addition, in the past 5 years, LAD has participated as a paid consultant for one-time engagements with Caris, Lyndra, Genocea Biosciences, Illumina and Cell Design Labs.

J.R.B. is a consultant/advisory board member of BMS, Merck, Medimmune/AstraZeneca, Johnson and Johnson, Syndax, Celgene, Amgen, EliLilly. JB received grant funding from BMS.

P.M.F. is a consultant/advisory board member of Abbvie, AstraZeneca, Bristol-Myers Squibb, Boehringer lngelheim, EMD Serono, Inivata, Janssen, Lilly, Merck, Novartis. PF received grant/research Support from AstraZeneca, Bristol-Meyers Squibb, Corvus, Kyowa, Novartis.

J.N. is a consultant/advisory board member for Bristol Myers Squibb, MedImmune/AstraZeneca, and Roche/ Genentech, has received research funding from Merck and MedImmune/AstraZeneca, and has received honoraria from Bristol Myers Squibb and MedImmune/AstraZeneca.

J.M.T. is consultant/advisory board member for Bristol-Myers Squibb, Astra Zeneca, and Merck. J.M.T. receives research funding from Bristol-Myers Squibb.

R.A.A. is a consultant/advisory board member for Bristol Myers Squibb, Merck, AstraZeneca, Adaptive Biotechnologies and has received research funding from Bristol Myers Squibb, Stand-up to Cancer and contractual work from Five Prime Therapeutics and FLX Bio.

C.L.S., F.H., N.J.L. receive research grant support from Bristol-Myers Squibb. N.J.L.: Master Nonclinical Research Agreement between Johns Hopkins University and Bristol-Myers Squibb Company; Pediatric Advisory Council BMS.

\section{Author details}

${ }^{1}$ Bloomberg-Kimmel Institute for Cancer Immunotherapy, Johns Hopkins University, Baltimore, MD, USA. ${ }^{2}$ Sidney Kimmel Comprehensive Cancer Center, Johns Hopkins University, Baltimore, MD, USA. ${ }^{3}$ Department of Pathology, Johns Hopkins University, Baltimore, MD, USA. ${ }^{4}$ Russell H. Morgan Department of Radiology and Radiological Science, Johns Hopkins University, Baltimore, MD, USA. ${ }^{5}$ Division of Biostatistics and Bioinformatics, Johns
Hopkins University, Baltimore, MD, USA. ${ }^{6}$ The Swim Across America Laboratory, John Hopkins University, Baltimore, MD, USA. 'Ludwig Center and Howard Hughes Medical Institute, Johns Hopkins University, Baltimore, MD, USA. ${ }^{8}$ Immunitrack, Copenhagen, Denmark. ${ }^{9}$ Department of Medicine, Division of Solid Tumor Oncology, Memorial Sloan Kettering Cancer Center, New York, NY, USA. ${ }^{10}$ Present address: B.R.B., Bioinformatics Core, Department of Complementary \& Integrative Medicine, University of Hawaii John A. Burns School of Medicine, Honolulu, HI 96813, USA.

Received: 26 February 2019 Accepted: 26 February 2019

Published online: 06 March 2019

\section{Reference}

1. Smith et al. (2019) Persistent mutant oncogene specific T cells in two patients benefitting from anti-PD-1 (2019) 7:40. https://doi.org/10.1186/ s40425-018-0492-X 